\title{
Breathing Pattern Characterization in Chronic Heart Failure Patients Using the Respiratory Flow Signal
}

\author{
A. Garde, ${ }^{1,2,3}$ L. Sörnmo, ${ }^{4,5}$ R. Jané, ${ }^{1,2,3}$ and B. F. Giraldo ${ }^{1,2,3}$ \\ ${ }^{1}$ Department ESAII, Universitat Politècnica de Catalunya (UPC), Pau Gargallo, 5, 08028 Barcelona, Spain; ${ }^{2}$ Institut de \\ Bioenginyeria de Catalunya (IBEC), Barcelona, Spain; ${ }^{3}$ CIBER de Bioingeniería, Biomateriales y Nanomedicina (CIBER-BBN), \\ Barcelona, Spain; ${ }^{4}$ Department of Electrical and Information Technology, Lund University, Lund, Sweden; and ${ }^{5}$ Center of \\ Integrative Electrocardiology, Lund University, Lund, Sweden
}

(Received 27 January 2010; accepted 18 June 2010; published online 8 July 2010)

Associate Editor John H. Linehan oversaw the review of this article.

\begin{abstract}
This study proposes a method for the characterization of respiratory patterns in chronic heart failure $(\mathrm{CHF})$ patients with periodic breathing (PB) and nonperiodic breathing (nPB), using the flow signal. Autoregressive modeling of the envelope of the respiratory flow signal is the starting point for the pattern characterization. Spectral parameters extracted from the discriminant frequency band (DB) are used to characterize the respiratory patterns. For each classification problem, the most discriminant parameter subset is selected using the leave-one-out cross-validation technique. The power in the right DB provides an accuracy of $84.6 \%$ when classifying $\mathrm{PB}$ vs. nPB patterns in $\mathrm{CHF}$ patients, whereas the power of the DB provides an accuracy of $85.5 \%$ when classifying the whole group of $\mathrm{CHF}$ patients vs. healthy subjects, and $85.2 \%$ when classifying $\mathrm{nPB}$ patients vs. healthy subjects.
\end{abstract}

Keywords-Chronic heart failure, AR modeling, Respiratory pattern, Discriminant band, Periodic and nonperiodic breathing.

\section{INTRODUCTION}

Patients with chronic heart failure (CHF) often develop breathing abnormalities, including various forms of oscillatory breathing patterns characterized by rises and falls in ventilation. ${ }^{2,22}$ Periodic breathing (PB) during sleep or wakefulness has been found to be a powerful predictor of poor prognosis in $\mathrm{CHF}$ patients. ${ }^{9} \mathrm{~PB}$ patterns can be classified into ventilation with apnea, commonly known as Cheyne-Stokes respiration (CSR), or ventilation without apnea., ${ }^{4,11}$ Recent studies report a PB prevalence as high as $70 \%$ in CHF patients. ${ }^{13}$ Moreover PB, and especially CSR,

Address correspondence to B. F. Giraldo, Department ESAII, Universitat Politècnica de Catalunya (UPC), Pau Gargallo, 5, 08028 Barcelona, Spain. Electronic mail: Beatriz.Giraldo@upc.edu has been associated with increased mortality. ${ }^{3,7,14}$ Accurate risk stratification is crucial both for establishing prognosis and appropriate allocation of limited resources for advanced, but expensive, treatments such as heart transplantation. ${ }^{1}$ Physiological parameters for the characterization and detection of different breathing patterns have been suggested in a number of clinical studies. ${ }^{16,18}$ The patterns are influenced by the degree of wakefulness, posture, and physiological and mental activity. ${ }^{15}$

There are multiple clinical predictors of outcome for heart failure patients such as the New York Heart Association (NYHA) classification stages, left ventricular ejection fraction, systolic arterial pressure, and peak $\mathrm{VO}_{2}$ at cardiopulmonary exercise testing, low exercise tolerance, arrhythmias, cardiothoracic ratio, and disturbances in the autonomic nervous system. Although these accepted risk indices perform well in the prediction of mortality due to disease progression, the prediction of sudden cardiac death (SCD) appears more problematic. In hospitalized, symptomatic CHF patients with high risk of all-cause mortality, death is often due to progressive pump failure. However, in ambulatory patients with less severe CHF, symptoms may be less obvious and death is more often sudden in nature. Therefore, it is important to identify mildly symptomatic $\mathrm{CHF}$ patients at risk for SCD. A combination of various risk markers that account for different information appears to be a better approach to predict the risk level in a heterogeneous CHF population. Thus, in view of the breathing abnormalities presented by CHF patients, we have focused on extracting relevant information from the respiratory pattern, which can be used to discriminate mildly symptomatic $\mathrm{CHF}$ patients from those at higher risk. 
Normal breathing cycle lengths range from 3 to $5 \mathrm{~s}$ (i.e., $0.20-0.33 \mathrm{~Hz}$ ). PB patterns have cycle lengths from 25 to $100 \mathrm{~s}$ (i.e., $0.01-0.04 \mathrm{~Hz}$ ). ${ }^{15}$ However, the same patient often exhibits a mixture of breathing patterns, ranging from nonperiodic breathing (nPB), i.e., without cyclic modulation of ventilation, through mild PB to CSR patterns. The origin of the PB pattern is still a matter of debate among researchers. The respiratory modulation frequency appears to be essential for the understanding of periodic and nPB patterns in CHF patients. Our previous studies were focused on characterizing the frequency band determined by the peak of the power spectral density (PSD) associated with the envelope of the respiratory flow signal. ${ }^{5,6}$

The aim of this study is to characterize the respiratory flow signal in CHF patients and healthy subjects using the envelope. Based on autoregressive (AR) power spectral analysis of the envelope, the relevant discriminant band (DB) is determined from the location of the modulation frequency peak, and characterized by a number of spectral parameters. This article expands considerably on the initial results obtained with this approach and presented by Garde et al..$^{5,6}$

\section{DATASET}

Respiratory flow signals were recorded from 35 healthy volunteers ( 23 females, $27 \pm 7$ years, respiratory frequency $15.5 \pm 3.7$ breaths/min) and $26 \mathrm{CHF}$ patients ( 7 females, $65 \pm 9$ years, $19.6 \pm 3.4$ breaths/ min) at Santa Creu i Sant Pau Hospital, Barcelona, Spain. All subjects were studied according to a protocol approved by the local Ethics Committee. The respiratory flow signals were acquired using a pneumotachograph, consisting of a Datex-Ohmeda monitor with a Validyne Model MP45-1-871 VariableReluctance Transducer (Validyne Corp., Northridge, CA, USA). The pneumotachograph was connected to a mask. The signals were recorded at $250 \mathrm{~Hz}$ sampling rate and 12 bit resolution.

Prior to data acquisition, a few minutes of adaptation were allowed in order to make the subjects feel comfortable with the mask. The respiratory flow signals were acquired during $15 \mathrm{~min}$ in both $\mathrm{CHF}$ patients and healthy subjects. All subjects were seated and remained awake throughout acquisition.

According to clinical criteria, the CHF patients were classified into two groups: 8 patients with $\mathrm{PB}$ patterns (one female, $71 \pm 7$ years, $18.4 \pm 2.2$ breaths $/$ min, $71.4 \pm$ 10.8 beats $/ \mathrm{min}$ ) and 18 patients with $\mathrm{nPB}$ pattern (five females, $62 \pm 9$ years, $22.5 \pm 4.3$ breaths $/ \mathrm{min}, 76.2 \pm$ 10.9 beats $/ \mathrm{min})$. Within the PB group, three patients were classified as CSR (one female, $68 \pm 6$ years,
$21.7 \pm 4.2$ breaths $/ \mathrm{min}, 66.0 \pm 5.3$ beats $/ \mathrm{min}$ ) and five patients as $\mathrm{PB}$ without apnea (no females, $73 \pm 8$ years, $23.0 \pm 4.7$ breaths $/ \mathrm{min}, \quad 81.0 \pm 9.2$ beats $/ \mathrm{min}$ ). $\mathrm{nPB}$ patients have 2-3 NYHA classification and $\mathrm{PB}$ patients have 3-4 NYHA classification. All patients show a decompensate heart activity. Figure 1 illustrates the different flow patterns observed in CSR, PB, nPB patients, and a healthy subject.

For the 61 subjects, the respiratory frequency was found to range from 0.2 to $0.4 \mathrm{~Hz}$, and the modulation frequency from 0.01 to $0.04 \mathrm{~Hz}$. These frequency ranges are in good agreement with those earlier reported by Pinna et al. ${ }^{15}$

\section{METHODS}

\section{Signal Preprocessing}

The respiratory flow signal is preprocessed with respect to artifact reduction in order to ensure robust signal analysis. Such processing is essential as certain artifacts can be difficult to distinguish from a normal breath. Outlier samples are rejected whose amplitude fall below the 1st percentile or exceed the 99th percentile of the entire signal's amplitude histogram; the outliers are replaced with the proper percentile value corresponding to 1 st or 99 th percentile. Short-duration spike artifacts, not removed with the percentile test, are detected using an auxiliary signal which results from sampling rate decimation of the original flow signal to $25 \mathrm{~Hz}$ followed by median filtering (a filter length of 11 was found suitable). Whenever the difference between the original and median-filtered signals exceeds a certain threshold value, here set to half the standard deviation of the signal, the original samples are replaced by the median value of the neighboring samples.

In a few patients, short gaps $(<1 \mathrm{~s})$ with missing samples occurred due to uncontrolled movements of the patients during acquisition. Rather than excluding these recordings from analysis, interpolation based on AR signal modeling was used to fill in the gaps. This method estimates the AR coefficients from the segment preceding the gap and then uses the coefficients to forward predict the signal across the missing samples. The same process is repeated for the segment that follows the gap using instead backward signal extrapolation. The gap of missing samples is then replaced by a crossfaded version of the two extrapolated values, using the following window ${ }^{8,20}$

$$
w(n)= \begin{cases}1-\frac{1}{2}(2 u(n))^{\alpha}, & u(n) \leq \frac{1}{2} \\ \frac{1}{2}(2-2 u(n))^{\alpha}, & u(n)>\frac{1}{2}\end{cases}
$$

where $u(n)=\left(n-n_{\mathrm{s}}\right) /\left(n_{\mathrm{e}}-n_{\mathrm{s}}\right)$, and $n_{\mathrm{s}}$ and $n_{\mathrm{e}}$ the indices of the onset and end of the gap, respectively. 

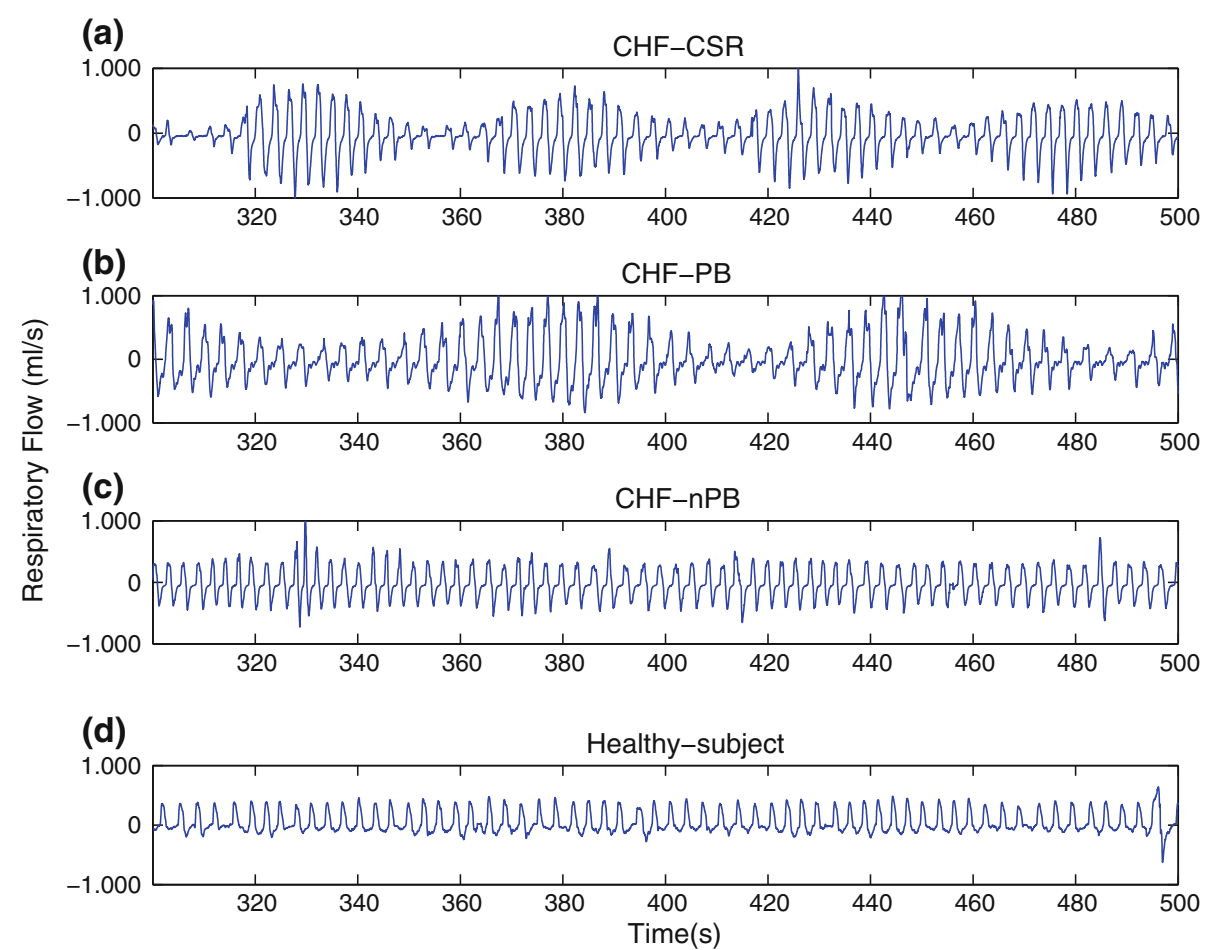

FIGURE 1. Excerpts of respiratory flow signals from a (a) CSR patient, (b) PB patient, (c) nPB patient, and (d) healthy subject.

Crossfading is carried out by multiplying the forward extrapolated sequence by $w(n)$ and the backward extrapolated sequence by $1-w(n)$. A linear downslope is attained with $\alpha=1$, whereas a step-like transition results when $\alpha \rightarrow \infty$. The slope of the window is adjusted via the parameter $\alpha=3$.

Finally, as the respiratory frequency does not exceed $0.5 \mathrm{~Hz}$, the respiratory flow signals are decimated to $1 \mathrm{~Hz}$, using appropriate low-pass filtering prior to downsampling. The decimation operation preserves the spectral information of interest, and ensures that AR modeling is not performed on an oversampled signal. $^{21}$

\section{Breathing Pattern Characterization}

The respiratory pattern characterization is based on the envelope of the respiratory flow signal, and thus contrasts previously described methods which are based on a sliding time window in which the amplitude, energy, or entropy are computed. ${ }^{10,12}$ For example, the normalized average Shannon energy, named as Shannon envelope, is a well-known technique which evaluates the average Shannon energy in successive signal segments. Nevertheless, there is a well-known technique ${ }^{21}$ based on the Hilbert transform (a 90-degree phase shifter) which extracts the envelope without the need of a sliding window. Figure 2 shows as an example of the preprocessed respiratory flow signal and the envelope of a $\mathrm{PB}$ patient. Considering the properties and performance of each envelope detection method, we choose the one based on Hilbert transform to extract the envelope of the respiratory flow signal.

A respiratory flow signal $s(n)$ may be modeled as an amplitude modulated low-pass signal

$$
s(n)=a(n) \cos \left(\omega_{\mathrm{m}} n+\phi\right)
$$

where $a(n), \omega_{\mathrm{m}}$, and $\phi$ denote the envelope, the modulation frequency, and the phase, respectively. It is well known that $a(n)$ can be retrieved by canceling negative frequencies and shifting the right-hand side spectrum to origin. ${ }^{17}$ The cancelation is accomplished by computing the analytical signal of $s(n)$, resulting from summation of $s(n)$ and its Hilbert transform $\bar{s}(n)$. Thus, the envelope is obtained without any prior knowledge of $\omega_{\mathrm{m}}$ and $\phi$, using

$$
a(n)=\sqrt{s^{2}(n)+\bar{s}^{2}(n)} .
$$

Since the bandwidth of $a(n)$ is much lower than $s(n)$, it can be decimated to $0.1 \mathrm{~Hz}$ without loss of information.

Due to the oscillatory character of respiratory flow signals, AR modeling is here employed for spectral analysis. The mean value of the envelope is subtracted and AR modeling is performed on the envelope signal. It is essential to have high spectral resolution in the 

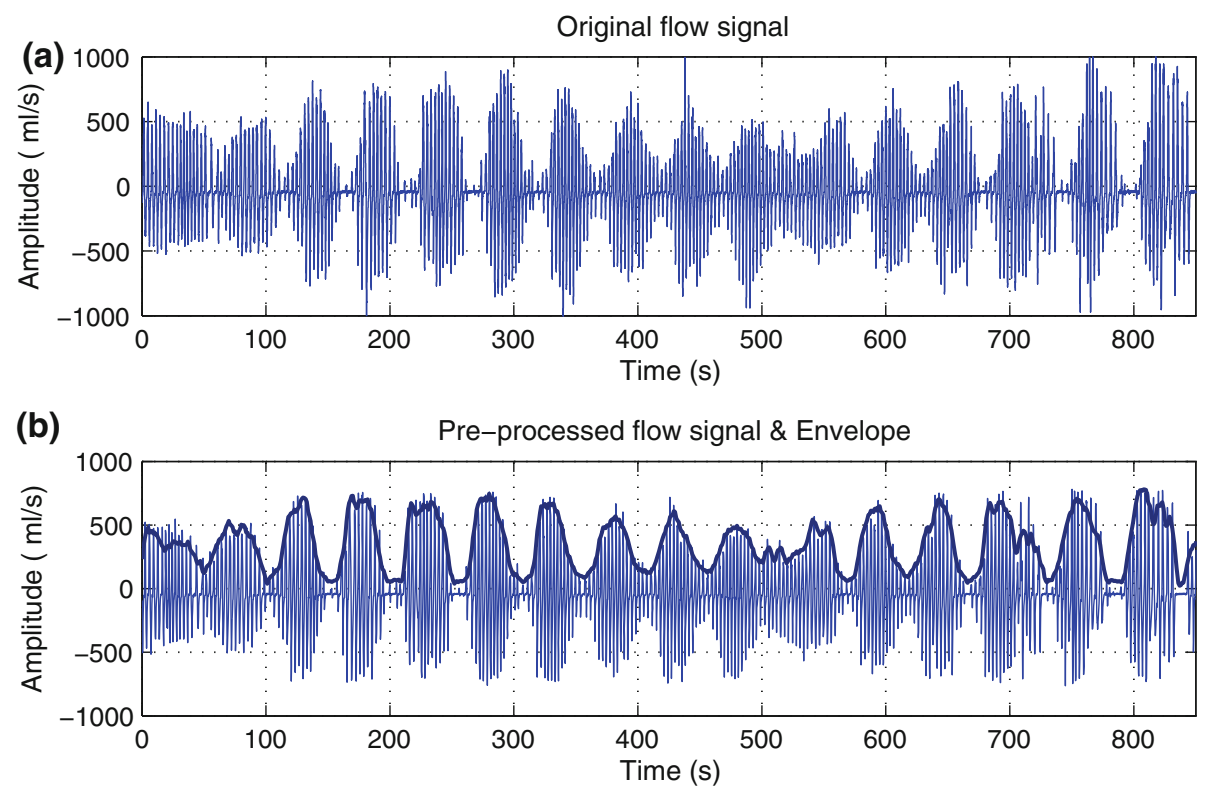

FIGURE 2. (a) Original flow signal, (b) preprocessed flow signal and its envelope, of a patient with PB pattern.

TABLE 1. Spectral parameters.

\begin{tabular}{lll}
\hline$P$ & Power in the DB & {$\left[f_{\mathrm{p}}-\Delta_{f} / 2, f_{\mathrm{p}}+\Delta_{f} / 2\right]$} \\
$P_{\mathrm{R}}$ & Power in the "right band" & {$\left[f_{\mathrm{p}}, f_{\mathrm{p}}+\Delta_{f} / 2\right]$} \\
$P_{\mathrm{L}}$ & Power in the "left band" & {$\left[f_{\mathrm{p}}-\Delta_{f} / 2, f_{\mathrm{p}}\right]$} \\
\hline
\end{tabular}

$f_{p}$, frequency peak; $\Delta_{f}$ frequency interval.

vicinity of the modulation peak, and therefore the following order selection procedure was adopted. First, the optimum order was determined in each subject using the minimum description length criterion. ${ }^{19}$ Then, the maximum of the resulting model order estimates was selected and used as global model order in the subsequent signal analysis. We apply the Ljung-Box statistical test to prove the whiteness of the prediction error.

\section{Parameter Extraction}

Certain spectral parameters are extracted from the DB defined by the frequency interval $\left(\Delta_{f}\right)$ centered at the modulation frequency peak $\left(f_{\mathrm{p}}\right)$ (Table 1$)$. Figure 3 shows the spectral parameters involve the power $(P)$, right side power $\left(P_{\mathrm{R}}\right)$, and left side power $\left(P_{\mathrm{L}}\right)$ of the DB.

Within the 61 subjects of this study, the respiratory frequency is found to range from 0.2 to $0.4 \mathrm{~Hz}$, whereas the modulation frequency ranged from 0.01 to $0.04 \mathrm{~Hz}$. Consequently, the modulating frequency peak $\left(f_{\mathrm{p}}\right)$ is searched for an interval ranging from 0.005 to $0.05 \mathrm{~Hz}$, i.e., slightly larger than the range of the modulation frequency. Having studied the frequency peaks presented by all subjects of the database, the frequency interval is fixed to $\Delta_{f}=0.02 \mathrm{~Hz}$. Figure 4 illustrates the different DBs for three CHF patients with different breathing patterns (CSR, PB without apnea, nPB) and a healthy subject.

\section{Data Analysis}

The accuracy of our pattern characterization is evaluated in terms of the following three classification problems: $\mathrm{CHF}$ patients vs. healthy subjects, nPB patients vs. healthy subjects, and CHF patients being either PB or $\mathrm{nPB}$.

First, the statistical significance of the abovementioned parameters derived from the DB is studied by the Mann-Whitney test. Next, a parameter selection process is implemented over the statistically significant parameters in order to select the most relevant subset (see Table 2). Leave-one-out crossvalidation technique is applied to the limited number of CHF patients (26 CHF patients and 35 healthy subjects). Only the most discriminative parameter is selected for each classification.

\section{RESULTS}

\section{Examples}

The global AR model order was found to be 4 when the above-mentioned selection procedure was applied. Applying the Ljung-Box test, the residuals of all 61 signals were found to be whitened by the global AR model. The present method is illustrated in Figs. 5-8 with examples taken from CSR, PB, nPB 


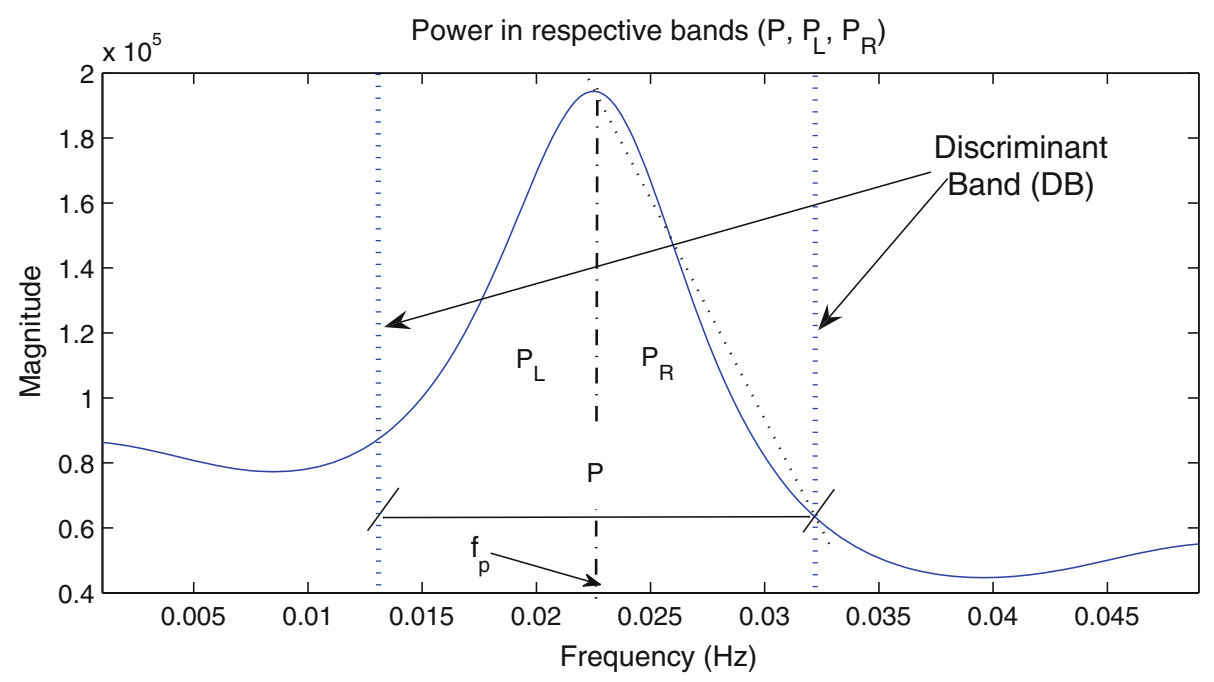

FIGURE 3. Spectral parameters extracted from the DB with a bandwidth of $\Delta_{f}=0.02 \mathrm{~Hz}$.

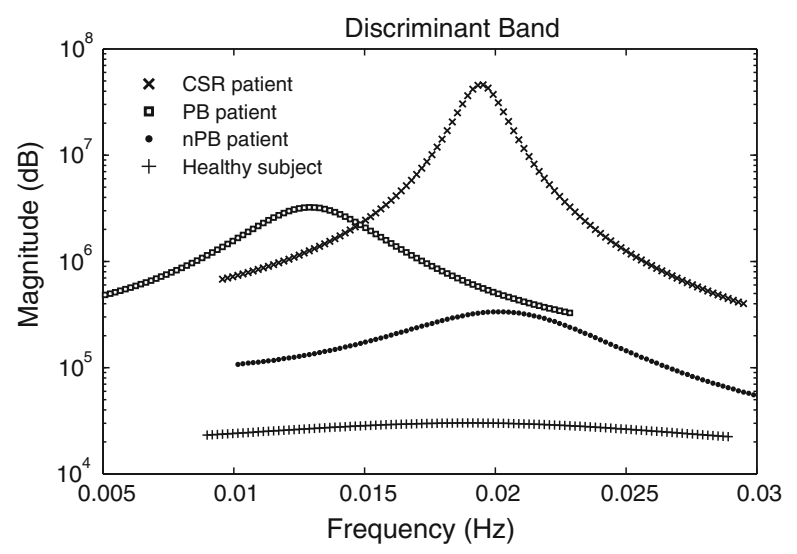

FIGURE 4. DB of a CSR patient, a PB patient without apnea, a nPB patient, and a healthy subject.

TABLE 2. $p$-Value of the statistically most significant parameters, for each classification.

\begin{tabular}{lccc}
\hline$p$-Value & $\begin{array}{c}\text { CHF vs. } \\
\text { healthy }\end{array}$ & $\begin{array}{c}\text { nPB-CHF vs. } \\
\text { healthy }\end{array}$ & PB vs. nPB (CHF) \\
\hline$P$ & $4.3 \times 10^{-9}$ & $7.3 \times 10^{-7}$ & $6.8 \times 10^{-4}$ \\
$P_{\mathrm{R}}$ & $1.3 \times 10^{-8}$ & $2.4 \times 10^{-6}$ & $4.6 \times 10^{-4}$ \\
$P_{\mathrm{L}}$ & $3.2 \times 10^{-9}$ & $5.2 \times 10^{-7}$ & $1.4 \times 10^{-3}$ \\
\hline
\end{tabular}

patients, and from a healthy subject. Figures 5 and 6 show that the PSDs of CSR and PB patients exhibit a clear frequency peak in the interval $0.005-0.03 \mathrm{~Hz}$. It is also obvious that respiratory modulation is more pronounced in the CSR patient than in the PB patient. As expected, the nPB patient and the healthy subject largely lack such modulation as the spectral peaks in this interval are much smaller (see Figs. 7 and 8 , respectively).

\section{Performance Evaluation}

Table 3 summarizes the results obtained with the most discriminant parameter for each of the three classification tasks.

The ROC curves of the statistically most significant parameters are studied so as to compare their performance in each classification. Table 4 presents the area under the curve (AUC) obtained with each parameter classifying different respiratory patterns. The AUC values validate the results obtained in all classifications with leave-one-out cross-validation technique. As expected, similar performance is obtained for the power-related parameters.

\section{DISCUSSION}

This article introduces envelope-based respiratory pattern characterization in $\mathrm{CHF}$ patients, used to quantify periodicity through spectral analysis. Our previous studies ${ }^{5,6}$ show that respiratory periodicity could be detected from the envelope of the flow signal using time-frequency analysis and employed for discriminating respiratory patterns in $\mathrm{CHF}$ patients. This article extends our previous work by investigating various envelope-based spectral parameters for identification of different respiratory patterns in $\mathrm{CHF}$ patients. It should be noted that the present approach to classification only involves one single parameter instead of three as our previous studies did. ${ }^{5,6}$

An advantage of the method is that outlier removal conditions the respiratory flow signals for robust envelope extraction. Although different methods have been developed to take out the respiratory flow 
(a)
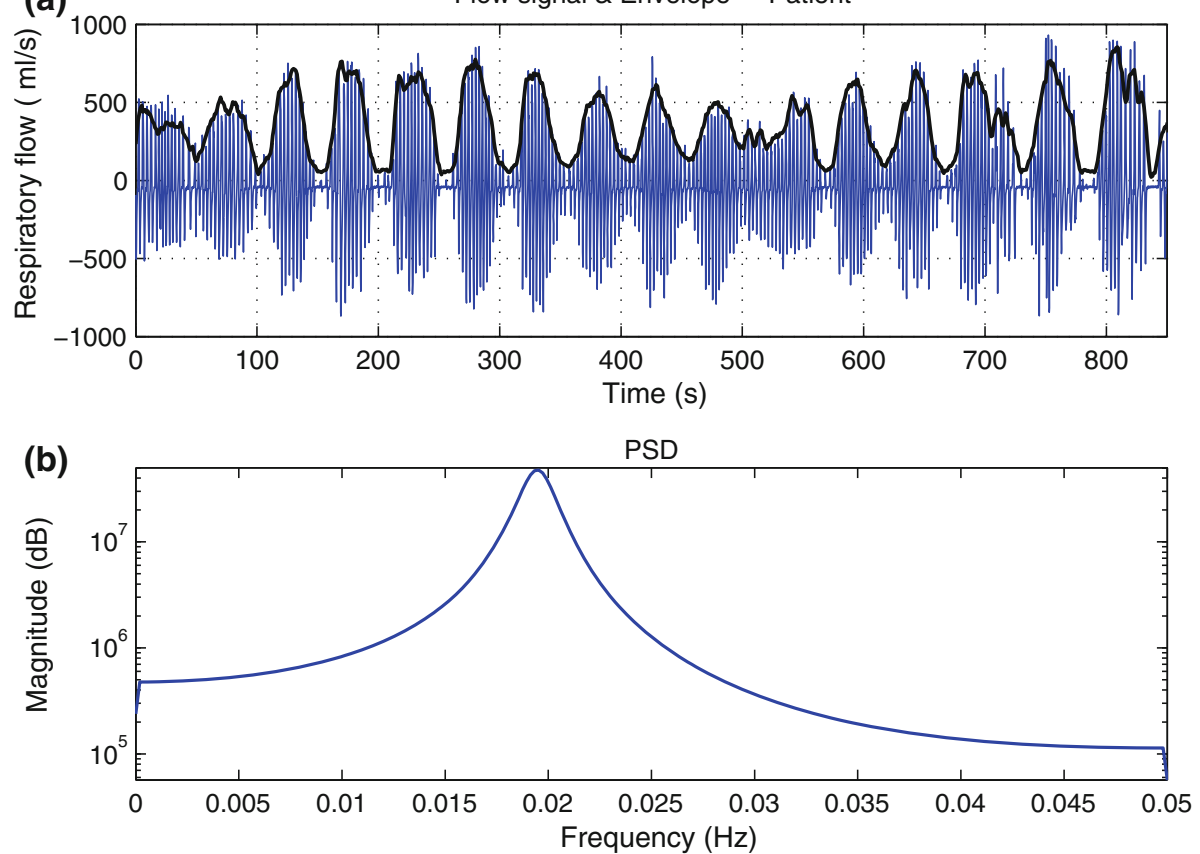

FIGURE 5. (a) The preprocessed flow signal and its envelope and (b) the PSD of a patient with CSR pattern.
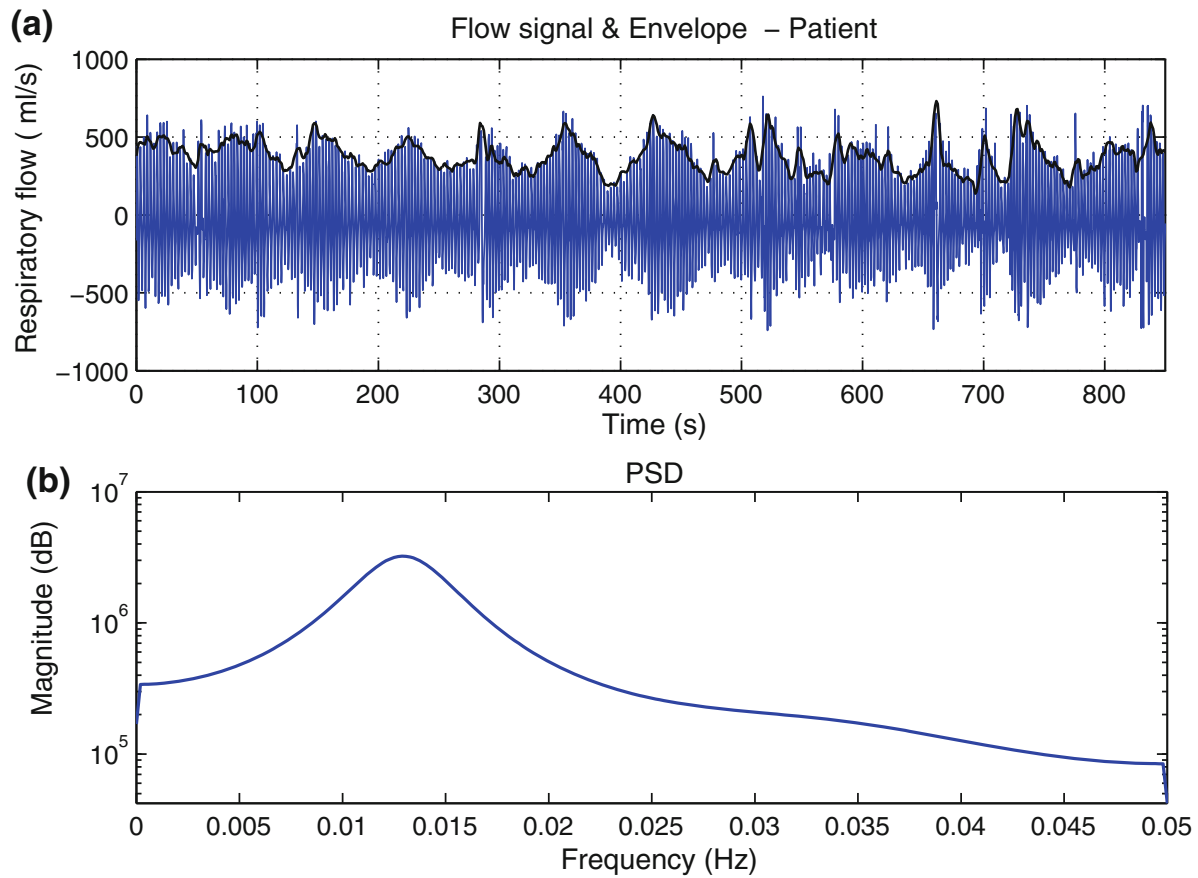

FIGURE 6. (a) The preprocessed flow signal and its envelope and (b) the PSD of a PB patient without apnea pattern.

envelope,$^{10}$ the one based on the Hilbert transform has presented good results and is the most efficient in terms of computational cost. Moreover, it does not require any normalization procedure which some of the other envelope extraction methods do.
The analysis of the respiratory flow signal provides a non-invasive tool to assess the autonomic nervous system influence on the respiratory pattern. Out of the established clinical predictors or linear HRV measures, the characterization of the respiratory flow signal 

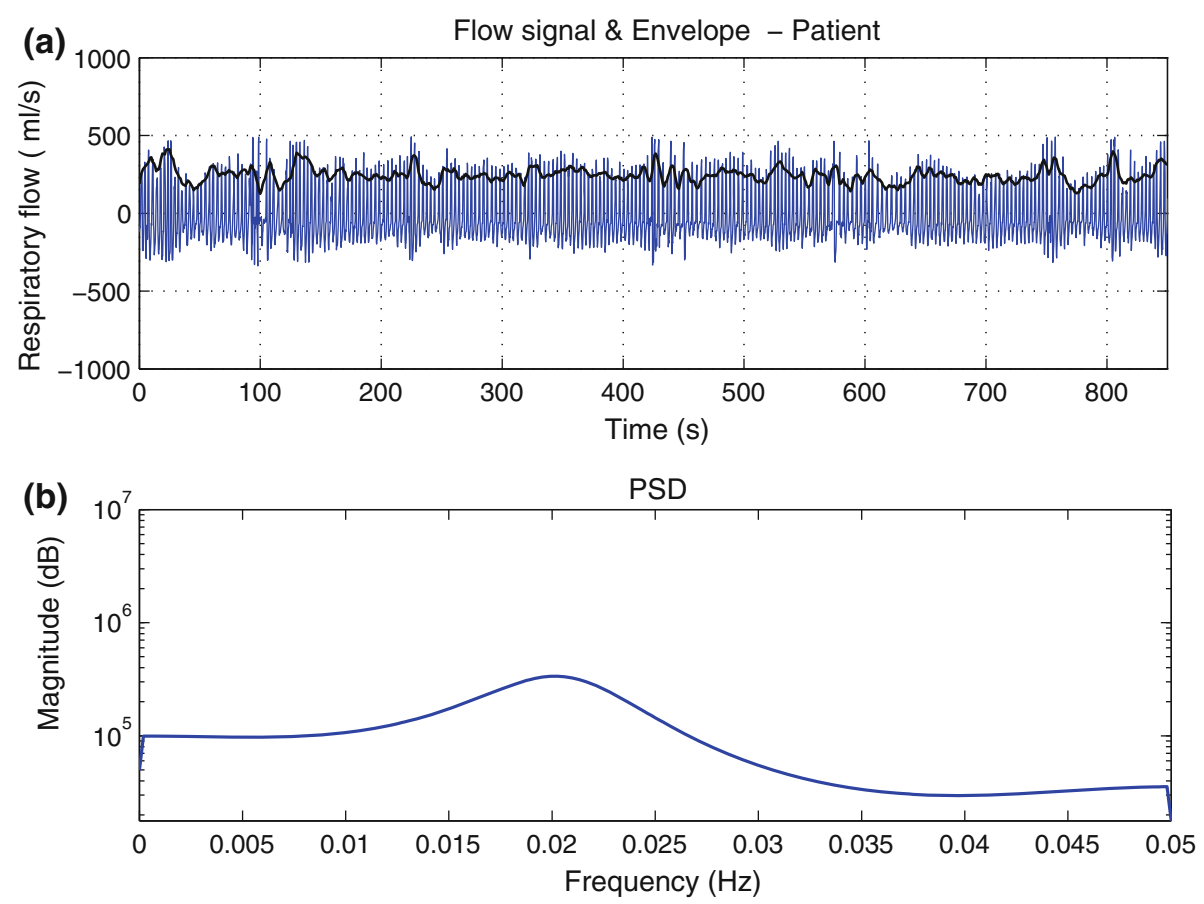

FIGURE 7. (a) The preprocessed flow signal and its envelope and (b) the PSD of an nPB patient.
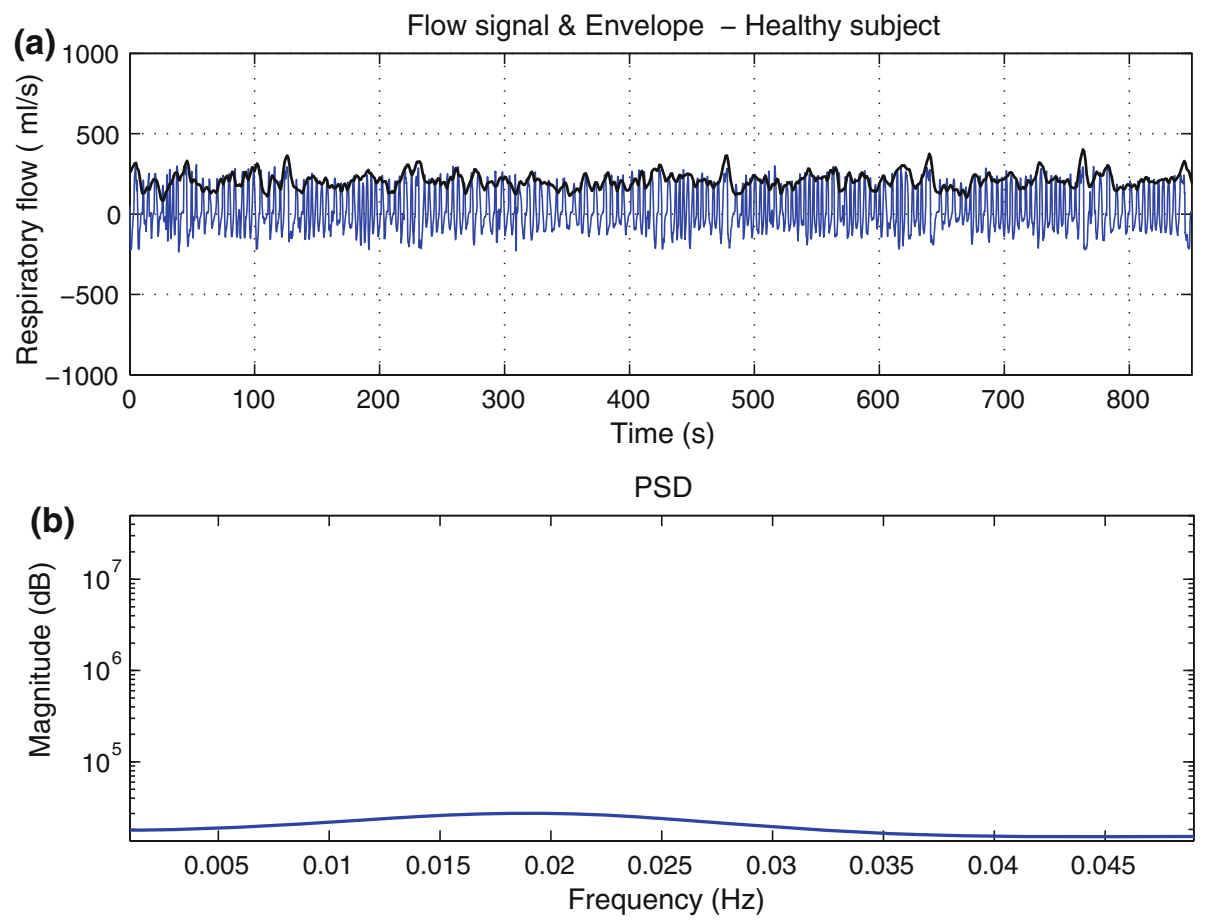

FIGURE 8. (a) The preprocessed flow signal and its envelope and (b) the PSD of a healthy subject.

envelope provides prediction markers to study the condition of $\mathrm{CHF}$ patients.

The main goal of this study is to characterize respiratory patterns that derive from $\mathrm{PB}$ and $\mathrm{nPB}$ patients, in order to identify different patterns and subsequently predict the condition of CHF patients. Both patient groups exhibit various degrees of periodicity depending on their condition, whereas healthy subjects have no pronounced periodicity. This observation is reflected by the parameter $P$ which decreases 
TABLE 3. Sensitivity (Sn), specificity (Sp), and total accuracy $(A c C)$, obtained with the best parameter for each classification with leave-one-out cross-validation technique.

\begin{tabular}{lcccc}
\hline Classification & Parameter & $S n(\%)$ & $S p(\%)$ & Acc (\%) \\
\hline CHF vs. healthy & $P$ & 85.2 & 85.7 & 85.5 \\
nPB-CHF vs. Healthy & $P_{\mathrm{L}}$ & 84.2 & 85.7 & 85.2 \\
PB vs. nPB (CHF) & $P_{\mathrm{R}}$ & 75.0 & 88.9 & 84.6 \\
\hline
\end{tabular}

TABLE 4. ROC area.

\begin{tabular}{lccc}
\hline$p$-Value & $\begin{array}{c}\text { CHF vs. } \\
\text { healthy (\%) }\end{array}$ & $\begin{array}{c}\text { nPB-CHF vs. } \\
\text { healthy (\%) }\end{array}$ & $\begin{array}{r}\text { PB vs. nPB } \\
(\text { CHF) }(\%)\end{array}$ \\
\hline$P$ & 93.8 & 91.1 & 92.1 \\
$P_{\mathrm{R}}$ & 92.4 & 89.2 & 93.4 \\
$P_{\mathrm{L}}$ & 94.1 & 91.7 & 89.5 \\
\hline
\end{tabular}

proportionally to the periodicity of the pattern. Due to their lack of modulation peak the healthy subjects show the smallest values for this parameter, providing promising results for discrimination, i.e., CHF vs. healthy $85.5 \%$, and nPB vs. healthy $85.2 \%$, despite the fact that $\mathrm{nPB}$ patients and healthy subjects present apparently similar respiratory patterns. The power of the right $\mathrm{DB}$ provided the best results when classifying $\mathrm{PB}$ and $\mathrm{nPB}$ breathing patterns into $\mathrm{CHF}$ patients. The ROC curves validate the results obtained when identifying different respiratory patterns.

The small size of the dataset is a limitation of this study, and therefore the significance of the results, though promising, needs to be further established on a larger set. As a consequence of the small size, it was decided to only investigate the performance of singleparameter linear classification.

The extracted parameters characterize the respiratory pattern of these patients, and suggest that $\mathrm{PB}$ patterns are related to the patients' condition. However, more research is needed to study the relevance of the information provided by these periodic respiratory patterns with respect to what can be extracted using the traditional clinical indices.

\section{CONCLUSIONS}

In this study, the respiratory flow envelope signal analysis is proposed for the characterization of respiratory flow signal patterns. Some spectral parameters are used to identify different respiratory patterns and classify $\mathrm{CHF}$ patients with different conditions and healthy subjects. Power-related parameters provide the best discrimination results between different respiratory patterns. Of the most discriminating parameters, the power of the modulation peak provided an accuracy of 85.5 and $85.2 \%$ classifying CHF patients vs. healthy subjects, and nPB patients vs. healthy subjects, and the power of the right DB showed an $84.6 \%$ of well-classified patients in an internal $\mathrm{CHF}$ patient classification between $\mathrm{PB}$ and $\mathrm{nPB}$ patterns. These results suggest that the method can be a useful tool to characterize different respiratory patterns in $\mathrm{CHF}$ patients and healthy subjects.

\section{ACKNOWLEDGMENTS}

This study was supported in part by Ministerio de Ciencia e Innovación under Grants TEC2007-68076C02-01 and TEC2007-63637 from the Spanish Government. The authors would like to thank Drs. S. Benito and A. Bayés-Genis and their collaborators of Santa Creu i Sant Pau Hospital, Barcelona, Spain, for their collaboration in the signal database acquisition.

\section{OPEN ACCESS}

This article is distributed under the terms of the Creative Commons Attribution Noncommercial License which permits any noncommercial use, distribution, and reproduction in any medium, provided the original author(s) and source are credited.

\section{REFERENCES}

${ }^{1}$ Chakko, S., and M. Gheorghiade. Estimating severity of chronic heart failure: a clinical challenge for the 1990. Am. Heart J. 124(1):260-264, 1992.

${ }^{2}$ Cherniack N. S., and G. S. Longobardo. Abnormalities in Respiratory Rhythm. Handbook of Physiology. The Respiratory System. Control of Breathing, vol. 2, chap. 22. Bethesda: American Physiological Society, pp. 729-749, 1986. ${ }^{3}$ Findley, L. J., C. W. Zwillich, S. Ancoli-Israel, D. Kripke, G. Tisi, and K. M. Moser. Cheyne Stokes breathing during sleep in patients with left ventricular heart failure. South. Med. J. 78(1):11-15, 1985.

${ }^{4}$ Francis, D. P., K. Willson, L. C. Davies, A. J. Coats, and M. Piepoli. Quantitative general theory for periodic breathing in chronic heart failure and its clinical implications. Circulation 102(18):2214-2221, 2000.

${ }^{5}$ Garde, A., B. Giraldo, R. Jan'e, I. Diaz, S. Herrera, S. Benito, M. Domingo, and A. Bayes-Genis. Analysis of respiratory flow signals in chronic heart failure patients with periodic breathing. In: Proceedings of the IEEE Conference of the Engineering in Medicine and Biology Society, pp. 307-310, 2007.

${ }^{6}$ Garde A., B. F. Giraldo, R. Jan'e, I. Diaz, S. Herrera, S. Benito, M. Domingo, and A. Bayes-Genis. Characterization of periodic and non-periodic breathing pattern in chronic heart failure patients. In: Proceedings of the IEEE Conference of the Engineering in Medicine and Biology Society, pp. 3227-3230, 2008.

${ }^{7}$ Hanly, P. J., and N. S. Zuberi-Khokhar. Increased mortality associated with Cheyne Stokes respiration in patients with congestive heart failure. Am. J. Respir. Crit. Care Med. 153(1):272-276, 1996. 
${ }^{8}$ Kauppinen, I., J. Kauppinen, and P. Saarinen. A method for long extrapolation of audio signals. J. Audio Eng. Soc. 49:1167-1180, 2001.

${ }^{9}$ Lanfranchi, P. A., A. Braghiroli, E. Bosimini, G. Mazzuero, R. Colombo, C. F. Donner, and P. Giannuzzi. Prognostic value of nocturnal Cheyne Stokes respiration in chronic heart failure. Circulation 99(11):1435-1440, 1999.

${ }^{10}$ Liang, H., S. Lukkarinen, and I. Hartimo. Heart sound segmentation algorithm based on heart sound envelogram. In: IEEE Proceedings of the Computers in Cardiology 1997, pp. 105-108, 1997.

${ }^{11}$ Lorenzi-Filho, G., P. R. Genta, A. C. Figueiredo, and D. Inoue. Cheyne-Stokes respiration in patients with congestive heart failure: causes and consequences. Clinics (Sao Paulo, Brazil) 60(4):333-344, 2005.

${ }^{12}$ Martínez-Alajarín, J., and R. Ruiz-Merino. Efficient method for events detection in phonocardiographic signals. Proc. SPIE 5839:398-409, 2005.

${ }^{13}$ Pinna, G. D., R. Maestri, A. Mortara, P. Johnson, T. Witkowski, P. Ponikowski, D. Andrews, S. Capomolla, M. T. La Rovere, and P. Sleight. Nocturnal periodic breathing is an independent predictor of cardiac death and multiple hospital admissions in heart failure. In: IEEE Proceedings of the Computers in Cardiology, vol. 33, pp. 837-840, 2006.

${ }^{14}$ Pinna, G. D., R. Maestri, A. Mortara, and M. T. La Rovere. Cardiorespiratory interactions during periodic breathing in awake chronic heart failure patients. Am. J. Physiol. Heart Circ. Physiol. 278(3):H932-H941, 2000.
${ }^{15}$ Pinna, G. D., R. Maestri, A. Mortara, M. T. La Rovere, F. Fanfulla, and P. Sleight. Periodic breathing in heart failure patients: testing the hypothesis of instability of the chemoreflex loop. J. Appl. Physiol. 89(6):2147-2157, 2000.

${ }^{16}$ Ponikowski, P., S. D. Anker, T. P. Chua, D. Francis, W. Banasiak, P. A. Poole-Wilson, A. J. Coats, and M. Piepoli. Oscillatory breathing patterns during wakefulness in patients with chronic heart failure: clinical implications and role of augmented peripheral chemosensitivity. Circulation 100(24):2418-2424, 1999.

${ }^{17}$ Proakis, J. G. Digital Communications (4th ed.). New Jersey: Prentice-Hall, 2001.

${ }^{18}$ Ribeiro, J. P. Periodic breathing in heart failure: bridging the gap between the sleep laboratory and the exercise laboratory. Circulation 113(1):9-10, 2006.

${ }^{19}$ Rissanen, J. Modeling by shortest data description. Automatica 14:465-471, 1978.

${ }^{20}$ Roth, K., I. Kauppinen, P. A. A. Esquef, and V. Valimaki. Frequency warped burg's method for ar-modeling. In: IEEE Workshop on Applications of Signal Processing to Audio and Acoustics, 2003, pp. 5-8, 2003.

${ }^{21}$ Sörnmo, L., and P. Laguna. Bioelectrical Signal Processing in Cardiac and Neurological Applications. Amsterdam: Elsevier/Academic Press, 2005.

${ }^{22}$ Yajima, T., A. Koike, K. Sugimoto, Y. Miyahara, F. Marumo, and M. Hiroe. Mechanism of periodic breathing in patients with cardiovascular disease. Chest 106(1):142146, 1994. 